\title{
ON TIME-LIKE RULED SURFACES IN MINKOWSKI 3-SPACE
}

\author{
CUMALI EKICI ${ }^{1}$, HAKAN ÖZTÜRK ${ }^{2, *}$ \\ ${ }^{1}$ DEPARTMENT OF MATHEMATICS AND COMPUTER SCIENCES, FACULTY OF SCIENCE AND ARTS, MEŞELIK \\ CAMPUS, ESKISSEHIR OSMANGAZI UNIVERSITY, 26480 ESKIŞEHIR,TURKEY \\ ${ }^{2}$ AFYON VOCATIONAL SCHOOL,ALI ÇETINKAYA CAMPUS, AFYON KOCATEPE UNIVERSITY, 03200 \\ AFYONKARAHISAR,TURKEY \\ ${ }^{*}$ CORRESPONDING AUTHOR: HOZTURK@AKU.EDU.TR
}

COPYRIGHT (C2013 HORIZON RESEARCH PUBLISHING ALL RIGHTS RESERVED.

\begin{abstract}
In this paper, a study of time-like ruled surfaces in Minkowski 3-space is investigated by strictly connected time-like straight line moving with Darboux's frame along a differentiable space-like curve. By using the striction curve and the distribution parameter of time-like ruled surfaces, some theorems related to the geodesic curvature and the second fundamental form tensor are obtained.
\end{abstract}

Keywords Time-like Ruled Surface, Second Fundamental Form Tensor, Geodesic Curvature, Distribution Parameter

M.S.C. 2000: 53A05, 53A 15

\section{INTRODUCTION}

Let $R^{3}$ be endowed with Lorentzian inner product of $X$ and $Y$ is defined by

$$
\langle X, Y\rangle=x_{1} y_{1}+x_{2} y_{2}-x_{3} y_{3},
$$

for $X, Y \in R^{3}$. $\left(R^{3},\langle\rangle,\right)$ is called Minkowski 3-space denoted by $R_{1}^{3}$. Then Lorentzian vector product of $X$ and $Y$ is defined by

$$
X \wedge Y=\left(x_{2} y_{3}-x_{3} y_{2}, x_{3} y_{1}-x_{1} y_{3}, x_{2} y_{1}-x_{1} y_{2}\right),
$$

for any vectors $X, Y \in R_{1}^{3}$, (see [8]).

The vectors $X \in R_{1}^{3}$ are called a space-like, time-like and null (light-like) vector if $\langle X, X\rangle>0$ or $X=0$, $\langle X, X\rangle<0$ and $\langle X, X\rangle=0$ for all $X \neq 0$, respectively. If $\langle X, Y\rangle=0$ for all $X$ and $Y$, the vectors $X$ and $Y$ are called orthogonal in the sense of Lorentzian. For $X \in R_{1}^{3}$, the norm of $X$ is defined by $\|X\|=\sqrt{|\langle X, X\rangle|}$ and $X$ is said to be a unit vector if $\|X\|=1$, (see [8]).

Let $\alpha(t): I \subset R \rightarrow R_{1}^{3}$, be a smooth regular curve in $R_{1}^{3}$. For any $t \in I$, the curve $\alpha$ is said to be a space-like, time-like and null curve if the velocity vector $\alpha^{\prime}(t)$ is a space-like, time-like or null vector, respectively, (see [8]).

A surface in Minkowski 3-space is called a time-like surface if the induced metric on the surface is a Lorentzian metric, i.e., the normal on the surface is a space-like vector. A time-like ruled surface in $R_{1}^{3}$ is obtained by a space-like straight line moving along a time-like curve or by a time-like straight line moving along a space-like curve. A time-like ruled surface is given by the parametrization

$$
\varphi: I \times R \rightarrow R_{1}^{3}, \varphi(t, v)=\alpha(t)+v X(t),
$$

in $R_{1}^{3}$, where the curve $\alpha$ is called the base curve and $X$ is called the ruling, (see [7]).

Let $\alpha=\alpha(s)$ be a unit speed space-like curve in $R_{1}^{3}$; The natural curvature and torsion of $\alpha(s)$ are denoted by $k_{1}(s), k_{2}(s)$, respectively. Consider the Frenet frame $\left\{V_{1}, V_{2}, V_{3}\right\}$ attached to the space-like curve $\alpha=\alpha(s)$ such that $V_{2}=V_{2}(s)$ is any principal normal vector field of type time-like, $V_{3}=V_{3}(s)$ is any binormal vector field of type space-like and $V_{1}=V_{1}(s)$ is the unit tangent vector field. Then the derivative formulas of Frenet frame $\left\{V_{1}, V_{2}, V_{3}\right\}$ can be written as

$$
\left[\begin{array}{l}
V_{1}^{\prime} \\
V_{2}^{\prime} \\
V_{3}^{\prime}
\end{array}\right]=\left[\begin{array}{ccc}
0 & k_{1} & 0 \\
k_{1} & 0 & k_{2} \\
0 & k_{2} & 0
\end{array}\right]\left[\begin{array}{l}
V_{1} \\
V_{2} \\
V_{3}
\end{array}\right]
$$

We also define Darboux frame attached to the space-like curve $\alpha=\alpha(s)$ such that $V_{1}=V_{1}(s)$ is a unit tangent vector field, $N=N(s)$ is a unit vector field of type space-like and $G=G(s)$ is a binormal vector field of type time-like defined by

$G(s)=V_{1}(s) \wedge N(s)$. So we obtain Darboux frame $\left\{V_{1}, G, N\right\}$ at the point $\alpha(s)$. Thus the derivative formulas of 
Darboux frame $\left\{V_{1}, G, N\right\}$ are written as follows:

$$
\begin{aligned}
V_{1}^{\prime}(s) & =k_{g} G(s)-k_{n} N(s) \\
G^{\prime}(s) & =k_{g} V_{1}(s)+\tau_{g} N(s) \\
N^{\prime}(s) & =k_{n} V_{1}(s)+\tau_{g} G(s),
\end{aligned}
$$

where $k_{g}, k_{n}$ and $\tau_{g}$ are defined by

$$
\begin{aligned}
k_{g} & =k_{1}(s) \cosh \varphi \\
k_{n} & =k_{1}(s) \sinh \varphi \\
\tau_{g} & =k_{2}(s)+\frac{d \varphi}{d s},
\end{aligned}
$$

and $\varphi$ is any hyperbolic angle between the time-like vectors $V_{2}$ and $G$, and called as geodesic curvature, normal curvature and geodesic torsion, respectively, (see [9]).

In this paper, we study some characteristic properties of time-like ruled surfaces related to the geodesic curvature and the second fundamental form tensor by the means of the information given above.

\section{Methodology}

Let $\alpha$ be a space-like curve with parameterized by arc length on a time-like ruled surface. In this situation, the derivative formulas of Darboux frame $\left\{V_{1}, G, N\right\}$ at the point $\alpha(s)$ can be written as

$$
\left[\begin{array}{c}
V_{1}^{\prime} \\
G^{\prime} \\
N^{\prime}
\end{array}\right]=\left[\begin{array}{ccc}
0 & k_{g} & -\left\langle\Pi\left(V_{1}, V_{1}\right), N\right\rangle \\
k_{g} & 0 & \left\langle\Pi\left(V_{1}, G\right), N\right\rangle \\
\left\langle\Pi\left(V_{1}, V_{1}\right), N\right\rangle & \left\langle\Pi\left(V_{1}, G\right), N\right\rangle & 0
\end{array}\right]\left[\begin{array}{c}
V_{1} \\
G \\
N
\end{array}\right],
$$

where $k_{g}$, $\Pi$ be the geodesic curvature and the second fundamental form tensor, respectively.

Now, we compute the distribution parameter of the time-like ruled surface $M$. Let $P_{X}$ be a distribution parameter of time-like ruling $X$. Then we have

$$
\begin{aligned}
P_{X} & =-\frac{\operatorname{det}\left(T, X, X^{\prime}\right)}{\left\|X^{\prime}\right\|^{2}} \\
& =\frac{\langle\Pi(T, X), N\rangle}{[\langle\Pi(T, X), N\rangle]^{2}+k_{g}^{2}},
\end{aligned}
$$

where $X^{\prime}$ is a time-like vector field.

Analogously, $P_{X}$ be a distribution parameter of space-like ruling $X$. So we get

$$
\begin{aligned}
P_{X} & =-\frac{\operatorname{det}\left(T, X, X^{\prime}\right)}{\left\|X^{\prime}\right\|^{2}} \\
& =\frac{\langle\Pi(T, X), N\rangle}{[\langle\Pi(T, X), N\rangle]^{2}-k_{g}^{2}},
\end{aligned}
$$

where $X^{\prime}$ is a space-like vector field.

Also, we obtain the striction curve of any time-like ruled surface $M$. If the distance between the base curve and the striction point of the time-like ruled surface $M$ is $\bar{u}$, then the position vector $\bar{\alpha}$ can be expressed in the form

$$
\bar{\alpha}(s, \bar{u})=\alpha(s)+\bar{u} X(s) .
$$

The parameter $\bar{u}$ can be expressed in terms of the position vector of the space-like directrix. So we get

$$
\begin{aligned}
\bar{u} & =-\frac{\left\langle T, X^{\prime}\right\rangle}{\left\langle X^{\prime}, X^{\prime}\right\rangle} \\
& =-\frac{k_{g}}{k_{g}^{2}+[\langle\Pi(T, X), N\rangle]^{2}} .
\end{aligned}
$$

Thus the striction curve is given by

$$
\bar{\alpha}(s, \bar{u})=\alpha(s)-\frac{k_{g}}{k_{g}^{2}+[\langle\Pi(T, X), N\rangle]^{2}} X(s) .
$$

Conversely, if $\left\langle X^{\prime}, X^{\prime}\right\rangle=0$, then the time-like ruled surface $M$ is a cylinder. For time-like ruled surfaces, the striction curve can be taken as directrix. Hence, we must take $\bar{u}=0$ in Eq. (4).

Now, we study neighborhood of a point $P$ in the time-like ruled surface $M$. We assume that time-like ruling $X$ is orthogonal to the directrix $\alpha$ at each point. Thus we can write $\langle T, X\rangle=0$, where $T$ is a tangent vector field of directrix $\alpha$. Let $N$ be a local unit normal for this coordinate neighborhood. So the unit vector fields $T, X$ and $N$ give orthonormal frame along the space-like curve $\alpha$ and we obtain Frenet frame for this system. Similarly, we 
can obtain Darboux frame $\left\{T, X^{*}, N^{*}\right\}$ at the same point. We can get the following derivative formulas of these frames $T, X$ and $N$,

$$
\left[\begin{array}{c}
D_{T} T \\
D_{T} X \\
D_{T} N
\end{array}\right]=\left[\begin{array}{ccc}
0 & k_{g} & -\langle\Pi(T, T), N\rangle \\
k_{g} & 0 & \langle\Pi(T, X), N\rangle \\
\langle\Pi(T, T), N\rangle & \langle\Pi(T, X), N\rangle & 0
\end{array}\right]\left[\begin{array}{c}
T \\
X \\
N
\end{array}\right] .
$$

Taking $v$ as constant, we get a space-like curve $\varphi_{v}=\alpha^{\prime}(s)+v X^{\prime}(s)$ on $M$ with tangent

$$
A=\left(1+k_{g} v\right) T+v\langle\Pi(T, X), N\rangle N .
$$

Hence, we can say that the space-like vector field $A$ is normal to $X$.

Methodologically, we investigate on a time-like ruled surfaces in two different ways. Firstly, we examine on a time-like ruled surfaces with time-like rulings. Then similiar calculations are performed using time-like directrix.

\section{Results}

In this section, we firstly study on a time-like ruled surface swept out by a time-like straight line $X$ moving along a differentiable space-like curve $\alpha$.

Let us consider $M$ as a time-like ruled surface in $R_{1}^{3}$. There always exists Frenet and Darboux frames at each point of a time-like curve $\alpha$ on this surface. If we coincide these frames at the point $P$, we get Eq. (7). Thus the following theorems can be given

Theorem 3.1. Let $M$ be a time-like ruled surface in $R_{1}^{3}$. The tangent planes of $M$ along a ruling are the same if and only if $\Pi(T, X)=0$.

Proof. Since the tangent space along a ruling is uniquely spanned by the system $\{A, X\}$, the space-like vector field $N$ is constant along a space-like ruling. Thus the system $\{A, T\}$ is linearly dependent. Let $v$ be a constant. Then we get a space-like curve $\varphi_{v}=A=\alpha^{\prime}(s)+v X^{\prime}(s)$ on $M$ with tangent

$$
A=\left(1+k_{g} v\right) T+v\langle\Pi(T, X), N\rangle N,
$$

where $A$ is a space-like vector field and $N \neq 0$. By the meaning of Eq. (8), we obtain $\Pi(T, X)=0$, which completes the proof.

Theorem 3.2. Let $M$ be a time-like ruled surface in $R_{1}^{3}$. The time-like ruled surface $M$ is developable if and only if the distribution parameter of the time-like ruled surface is zero.

Proof. With the help of Eq. (2) and Theorem 3.1, we find $P_{X}=0$.

Theorem 3.3. Let $M$ be a time-like ruled surface in $R_{1}^{3}$, where $\Pi(T, X) \neq 0$. The shortest distance between the rulings of time-like ruled surfaces $M$ along the orthogonal trajectories is the distance measured by the value $v=-\frac{k_{g}}{k_{g}^{2}+[\langle\Pi(T, X), N\rangle]^{2}}$ along the space-like curve $\varphi_{v}: I \rightarrow M$.

Proof. We consider two rulings passing at the points $\alpha\left(s_{0}\right)$ and $\alpha\left(s_{1}\right)$, where $s_{0}<s_{1}$. The distance between these rulings along an orthogonal trajectory is given by

$$
J(v)=\int_{s_{0}}^{s_{1}}\|A\| d s
$$

where $A$ is a space-like vector field. Using Eq. (8), we get

$$
J(v)=\int_{s_{0}}^{s_{1}} \sqrt{1+2 v k_{g}+k_{g}^{2} v^{2}+v^{2}[\langle\Pi(T, X), N\rangle]^{2}} d s .
$$

To estimate the minimizing the value of $J(v)$, we use the derivative function of $J(v)$. So we conclude that

completing the proof.

$$
v=-\frac{k_{g}}{k_{g}^{2}+[\langle\Pi(T, X), N\rangle]^{2}},
$$

Theorem 3.4. Let $M$ be a non developable time-like ruled surface in $R_{1}^{3}$. Any point $\varphi\left(s, v_{0}\right)$ is a striction point on the main ruling passing the point $\alpha(s)$ if and only if $D_{T} X$ is the unit normal vector field of tangent plane in the point $\varphi\left(s, v_{0}\right)$, where $X$ is the tangent vector field of the space-like directrix $\alpha$ and $v_{0} \in R$.

Proof. Let any point $\varphi\left(s, v_{0}\right)$ be a striction point on the main ruling passing at the point $\alpha(s)$. We must show that $\left\langle D_{T} X, A\right\rangle=0$. Since $\langle X, X\rangle=-1$, we obtain $\left\langle D_{T} X, X\right\rangle=0$. Also, since $A=\varphi_{v}$ and $X$ be the tangent vector,then we have the following equation

$$
\left\langle D_{T} X, A\right\rangle=k_{g}+v_{0}\left[k_{g}^{2}+[\langle\Pi(T, X), N\rangle]^{2}\right] .
$$


Since $\varphi\left(s, v_{0}\right)$ is a striction point, we have

$$
v_{0}=-\frac{k_{g}}{k_{g}^{2}+[\langle\Pi(T, X), N\rangle]^{2}}
$$

Then substituting the value $v_{0}=-\frac{k_{g}}{k_{g}^{2}+[\langle\Pi(T, X), N\rangle]^{2}}$ into Eq. (9), we obtain $\left\langle D_{T} X, A\right\rangle=0$, which means that $D_{T} X$ is normal to $X$.

Conversely, the tangent vector field of the time-like curve $\varphi_{v_{0}}: I \rightarrow M$ is given

$$
A=\left(1+k_{g} v_{0}\right) T+v_{0}\langle\Pi(T, X), N\rangle N .
$$

Since $D_{T} X$ is normal to the tangent plane, we get

$$
v_{0}=-\frac{k_{g}}{k_{g}^{2}+[\langle\Pi(T, X), N\rangle]^{2}}
$$

Therefore, the point $\varphi\left(s, v_{0}\right)$ is a striction point on a main ruling.

Theorem 3.5. Let $M$ be a non developable time-like ruled surface in $R_{1}^{3}$. The absolute value of Gaussian curvature function $K$ of the time-like ruled surface takes its maximum value at the striction point on each one of the rulings.

Proof. Let $X$ be a time-like vector field of which the rulings pass through the point $P \in M$. An orthogonal basis $\{X, Y\}$ can be obtained, where $Y$ is a space-like vector field. Let $S$ be the matrix corresponding to the shape operator of $M$ derived from $N$. Also, the Gaussian curvature function is defined as

$$
K(s, v)=\operatorname{det} S=[\langle S(X), Y\rangle]^{2} .
$$

Since $A=\varphi_{v},\left\{A^{*}, X\right\}$ is an orthonormal basis of $\chi(M)$, where $A^{*}=\frac{A}{\|A\|}$. Hence, we obtain

$$
K(s, v)=\left[\left\langle S\left(A^{*}\right), X\right\rangle\right]^{2}=\frac{[\langle\Pi(T, X), N\rangle]^{2}}{\left[1+2 k_{g} v+v^{2}\left(\langle\Pi(T, X), N\rangle^{2}+k_{g}^{2}\right)\right]^{2}} .
$$

Differentiating Eq. (11) with respect to $v$, we get

$$
\frac{\partial K(s, v)}{\partial v}=-4 \frac{\langle\Pi(T, X), N\rangle^{2}\left[\left(\langle\Pi(T, X), N\rangle^{2}-k_{g}^{2}\right) v-k_{g}\right]}{\left[-\left(1+k_{g} v\right)^{2}+v^{2}\langle\Pi(T, X), N\rangle^{2}\right]^{3}} .
$$

So the value of $v=-\frac{k_{g}}{k_{g}^{2}+[\langle\Pi(T, X), N\rangle]^{2}}$ gives the maximum of $K(s, v)$ since

$$
\frac{\partial^{2} K(s, v)}{\partial v^{2}}\left(\frac{k_{g}}{[\langle\Pi(T, X), N\rangle]^{2}-k_{g}^{2}}\right)<0
$$

Hence, Gaussian curvature function has the maximum value at the striction point on each one of the rulings since the striction point corresponds to the value

$$
v=-\frac{k_{g}}{k_{g}^{2}+[\langle\Pi(T, X), N\rangle]^{2}} .
$$

Then using Eq. (10), we find

$$
K_{\max }(v)=\frac{\left[[\langle\Pi(T, X), N\rangle]^{2}+k_{g}^{2}\right]^{2}}{[\langle\Pi(T, X), N\rangle]^{2}} .
$$

On the other hand, combining Eqs. (2) and (12), we obtain

since $k_{g}=0$ at the striction point. Therefore, we have

$$
\begin{aligned}
K_{\max }(v) & =[\langle\Pi(T, X), N\rangle]^{2} \\
& =\left(\frac{1}{P_{X}}\right)^{2},
\end{aligned}
$$

$$
P_{X}=\frac{1}{\sqrt{K_{\max }(v)}} .
$$

The above relation means that the maximum value of the Gaussian curvature function of the time-like ruled surface $M$ along a ruling only depends on the distribution parameter. The value of $K_{\max }(v)$ is unique along a ruling which completes the proof. 
The important theorem concerning with the striction point of any ruled surfaces in Euclidean 3-space was given by Chasles (see [1], [3] ). Now, we can give this theorem for time-like ruled surfaces in Minkowski 3-space related to the second fundamental form tensor as follows:

Theorem 3.6. Let $M$ be a non developable time-like ruled surface in $R_{1}^{3}$ and $\theta$ be the angle between the normal vector at the point of a ruling and the normal vector at the striction point of this ruling. Then the tan $\theta$ is proportional to the distance between these points and the coefficient of proportionality is the inverse of the distribution parameter.

Proof. Let the point $\varphi(s, 0)$ be a striction point of $M$ for $v=0$. The curve of $\alpha: I \rightarrow M$ is an orthogonal trajectory which passes through the striction point $\varphi(s, 0)$. Then $D_{T} X$ is normal vector at $v=0$ and $k_{g}=0$. Thus the distribution parameter $P_{X}$ is written as follows:

$$
P_{X}=\frac{1}{\langle\Pi(T, X), N\rangle} .
$$

Also, the normal vector $N_{v}$ along the ruling is given by

$$
N_{v}=\frac{-\langle\Pi(T, X), N\rangle v T+N}{\sqrt{1+\langle\Pi(T, X), N\rangle^{2} v^{2}}} .
$$

On the other hand, $N$ and $N_{v}$ are the unit space-like vectors. Let $\theta$ be the angle between $N$ and $N_{v}$. Therefore, we obtain

Thus we have

$$
\left\langle N, N_{v}\right\rangle=\frac{1}{\sqrt{1+\langle\Pi(T, X), N\rangle^{2} v^{2}}} .
$$

$$
\cos \theta=\frac{1}{\sqrt{1+\langle\Pi(T, X), N\rangle^{2} v^{2}}} .
$$

By the means of Eq. (2), we obtain

$$
\cos \theta=\frac{1}{\sqrt{1+\left(\frac{v}{P_{X}}\right)^{2}}},
$$

which implies

$$
\tan \theta=\frac{v}{P_{X}}
$$

This is the desired result.

Now, we study on a time-like ruled surface swept out by a space-like straight line $X$ moving along a differentiable time-like curve $\alpha$.

We consider $M$ is a time-like ruled surface in $R_{1}^{3}$. There exists Frenet and Darboux frames at each point of a time-like curve $\alpha$ on this surface. If we coincide these frames at the point $P$, we obtain

$$
\left[\begin{array}{c}
D_{T} T \\
D_{T} X \\
D_{T} N
\end{array}\right]=\left[\begin{array}{ccc}
0 & k_{g} & -\langle\Pi(T, T), N\rangle \\
k_{g} & 0 & \langle\Pi(T, X), N\rangle \\
-\langle\Pi(T, T), N\rangle & -\langle\Pi(T, X), N\rangle & 0
\end{array}\right]\left[\begin{array}{c}
T \\
X \\
N
\end{array}\right] .
$$

Thus the following theorems can be given:

Theorem 3.7. Let $M$ be a time-like ruled surface in $R_{1}^{3}$. The tangent planes of $M$ along a ruling are the same if and only if $\Pi(T, X)=0$.

Proof. Since the tangent space along a ruling is spanned by the system $\{A, X\}$. So this tangent space is unique along a ruling. Then the space-like vector field $N$ is constant along a time-like ruling $\alpha$. Hence, the system $\{A, T\}$ is linearly dependent. Let $v$ be a constant, then we get a time-like curve $\varphi_{v}=A=\alpha^{\prime}(s)+v X^{\prime}(s)$ on $M$ with tangent

$$
A=\left(1+k_{g} v\right) T+v\langle\Pi(T, X), N\rangle N,
$$

where $A$ is a time-like vector field. By using Eq. (14), we obtain $\Pi(T, X)=0$.

Theorem 3.8. Let $M$ be a time-like ruled surface in $R_{1}^{3}$. The time-like ruled surface $M$ is developable if and only if the distribution parameter of the time-like ruled surface is zero.

Proof. Proof follows from Eqs. (3) and Theorem 3.7.

Theorem 3.9. Let $M$ be a time-like ruled surface in $R_{1}^{3}$, where $\Pi(T, X) \neq 0$. The longest distance between the rulings of time-like ruled surface $M$ along the orthogonal trajectories is the distance measured by the value $v=\frac{k_{g}}{[\langle\Pi(T, X), N\rangle]^{2}-k_{g}^{2}}$ along the time-like curve $\varphi_{v}: I \rightarrow M$. 
Proof. We consider two rulings passing at the points $\alpha\left(s_{0}\right)$ and $\alpha\left(s_{1}\right)$, where $s_{0}<s_{1}$. The distance between these rulings along an orthogonal trajectory is given by

$$
J(v)=\int_{s_{0}}^{s_{1}}\|A\| d s
$$

where $A$ is a time-like vector field. Then using Eq. (14), we have

$$
J(v)=\int_{s_{0}}^{s_{1}} \sqrt{1+2 v k_{g}+k_{g}^{2} v^{2}-v^{2}[\langle\Pi(T, X), N\rangle]^{2}} d s .
$$

To find the value of $v$, which maximizes $J(v)$, we use the derivative function of $J(v)$. So we deduce

which completes the proof.

$$
v=\frac{k_{g}}{[\langle\Pi(T, X), N\rangle]^{2}-k_{g}^{2}},
$$

Theorem 3.10. Let $M$ be a non developable time-like ruled surface in $R_{1}^{3}$. Any point $\varphi\left(s, v_{0}\right)$ is a striction point on the main ruling passing at the point $\alpha(s)$ if and only if $D_{T} X$ is the unit normal vector field of tangent plane in the point $\varphi\left(s, v_{0}\right)$, where $X$ is the tangent vector field of the time-like directrix $\alpha$ and $v_{0} \in R$.

Proof. Let $\varphi\left(s, v_{0}\right)$ be a striction point on the main ruling passing at the point $\alpha(s)$. We must show that $\left\langle D_{T} X, A\right\rangle=$ 0 . If we take the derivative of equation $\langle X, X\rangle=1$, then we obtain $\left\langle D_{T} X, X\right\rangle=0$. By direct computation, the value $\left\langle D_{T} X, A\right\rangle$ is obtained as follows:

$$
\left\langle D_{T} X, A\right\rangle=-k_{g}+v_{0}\left[-k_{g}^{2}+[\langle\Pi(T, X), N\rangle]^{2}\right]
$$

Since $\varphi\left(s, v_{0}\right)$ is a striction point, we have

$$
v_{0}=\frac{k_{g}}{[\langle\Pi(T, X), N\rangle]^{2}-k_{g}^{2}} .
$$

Then substituting the value $v_{0}=\frac{k_{g}}{[\langle\Pi(T, X), N\rangle]^{2}-k_{g}^{2}}$ into Eq. (15), we obtain $\left\langle D_{T} X, A\right\rangle=0$. It means that $D_{T} X$ is normal to $X$.

Conversely, the tangent vector field of the time-like curve $\varphi_{v_{0}}: I \rightarrow M$ is given

$$
A=\left(1+k_{g} v_{0}\right) T+v_{0}\langle\Pi(T, X), N\rangle N .
$$

Since $D_{T} X$ is normal to the tangent plane, we also have

$$
v_{0}=\frac{k_{g}}{[\langle\Pi(T, X), N\rangle]^{2}-k_{g}^{2}} .
$$

Therefore, the point $\varphi\left(s, v_{0}\right)$ is a striction point on a main ruling which shows desired result.

Theorem 3.11. Let $M$ be a non developable time-like ruled surface in $R_{1}^{3}$. The absolute value of Gaussian curvature function $K$ of the time-like ruled surface takes its minimum value at the striction point on each one of the rulings.

Proof. Let $X$ be a space-like vector field of which the rulings through the point $P \in M$. An orthogonal basis $\{X, Y\}$ can be obtained, where $Y$ is a time-like vector field. Let $S$ be the matrix corresponding to the shape operator of $M$ derived from $N$. Then Gaussian curvature function is given by

$$
K(s, v)=\operatorname{det} S=[\langle S(X), Y\rangle]^{2} .
$$

$\left\{A^{*}, X\right\}$ is an orthonormal basis of $\chi(M)$, where $A^{*}=\frac{A}{\|A\|}$. Hence, we obtain

$$
K(s, v)=\left[\left\langle S\left(A^{*}\right), X\right\rangle\right]^{2}=\frac{[\langle\Pi(T, X), N\rangle]^{2}}{\left[-1-2 k_{g} v+v^{2}\left(\langle\Pi(T, X), N\rangle^{2}-k_{g}^{2}\right)\right]^{2}} .
$$

Differentiating Eq. (17) with respect to $v$, we get

$$
\frac{\partial K(s, v)}{\partial v}=-4 \frac{\langle\Pi(T, X), N\rangle^{2}\left[\left(\langle\Pi(T, X), N\rangle^{2}-k_{g}^{2}\right) v-k_{g}\right]}{\left[-\left(1+k_{g} v\right)^{2}+v^{2}\langle\Pi(T, X), N\rangle^{2}\right]^{3}} .
$$

Thus the value of $v=\frac{k_{g}}{[\langle\Pi(T, X), N\rangle]^{2}-k_{g}^{2}}$ gives the minimum of $K(s, v)$, since

$$
\frac{\partial^{2} K(s, v)}{\partial v^{2}}\left(\frac{k_{g}}{[\langle\Pi(T, X), N\rangle]^{2}-k_{g}^{2}}\right)>0
$$


Hence, Gaussian curvature function has the minimum value at the striction point on each one of the rulings, since a striction point corresponds to the value

$$
v=\frac{k_{g}}{[\langle\Pi(T, X), N\rangle]^{2}-k_{g}^{2}} .
$$

With the help of Eq. (17), we have

$$
K_{\min }(v)=\frac{\left[[\langle\Pi(T, X), N\rangle]^{2}-k_{g}^{2}\right]^{2}}{[\langle\Pi(T, X), N\rangle]^{2}} .
$$

On the other hand, combining Eqs. (3) and (18), we obtain

$$
\begin{aligned}
K_{\min }(v) & =[\langle\Pi(T, X), N\rangle]^{2} \\
& =\left(\frac{1}{P_{X}}\right)^{2},
\end{aligned}
$$

since $k_{g}=0$ at the striction point. Therefore, we find

$$
P_{X}=\frac{1}{\sqrt{K_{\min }(v)}} .
$$

which means the minimum value of Gaussian curvature function of time-like ruled surface $M$ along a ruling only depends on the distribution parameter. The value of $K_{\min }(v)$ is unique along a ruling.

Theorem 3.12. Let $M$ be a non developable time-like ruled surface in $R_{1}^{3}$ and $\theta$ be an angle between the normal vector at the point of a ruling and the normal vector at the striction point of this ruling. Then $\tan \theta$ is proportional to the distance between these points and the coefficient of proportionality is the inverse of the distribution parameter.

Proof. Let the point $\varphi(s, 0)$ be a striction point of $M$ for $v=0$. The curve of $\alpha: I \rightarrow M$ is an orthogonal trajectory which passes through the striction point $\varphi(s, 0)$. Then $D_{T} X$ is normal vector at $v=0$ and $k_{g}=0$. Thus the distribution parameter $P_{X}$ is written as follows:

$$
P_{X}=\frac{1}{\langle\Pi(T, X), N\rangle} .
$$

Also, the normal vector $N_{v}$ along the ruling is given by

$$
N_{v}=\frac{\langle\Pi(T, X), N\rangle v T+N}{\sqrt{1-\langle\Pi(T, X), N\rangle^{2} v^{2}}} .
$$

On the other hand, $N$ and $N_{v}$ are the unit space-like vectors. Let $\theta$ be the angle between $N$ and $N_{v}$. Therefore, we obtain

$$
\left\langle N, N_{v}\right\rangle=\frac{1}{\sqrt{1-\langle\Pi(T, X), N\rangle^{2} v^{2}}} .
$$

Thus we get

$$
\cos \theta=\frac{1}{\sqrt{1-\langle\Pi(T, X), N\rangle^{2} v^{2}}} .
$$

By using Eq.(3), we obtain

$$
\cos \theta=\frac{1}{\sqrt{1-\left(\frac{v}{P_{X}}\right)^{2}}}
$$

Therefore, we have

$$
v=\tan \theta P_{X}
$$

which completes the proof.

\section{Discussion}

The theory of special relativity was created by Einstein in 1905. Then Poincare has shown that the transformation of this special relativity (The Lorentzian Transformation) is invariant of rotations in 3-dimensional Euclidean space. Following these studies, Minkowski investigated this analogy and he realized that space and time could be linked in 4-dimensional continuum, with coordinates $(x, y, z, i c t)$ where $c$ is the speed of light in a vacuum and the variable is $i=\sqrt{-1}$, called "pure imaginary". The Lorentzian transformations are no more than rotations in Minkowski space. The constancy of the speed of light can be understood by considering the emission of a light signal at any position and the reception of the same signal in a differerent position. Using comparing these two events, the equation of the speed of light is obtained and it can be interpreted as the expressing invariance of a null distance in a space with three real dimensions and one imaginary dimension. 
The space-time continuum provided a framework for all works in relativity. Einstein was developed a frame in general relativity. However, the simplier Minkowski space was not able to deal with accelerating reference frames and was to be replaced by the more versatile Riemannian space.

The purpose of this paper is to give an informal introduction to the geometry of 3-dimensinonal Minkowski space in the light of the parallelism with 3-dimensional Euclidean space. The well-known theorems in 3-dimensional Euclidean space are proved for a time-like ruled surface with time-like rulings or time-like directrix.

\section{Conclusion}

As revealed from the foregoing relations and theorems in section 3, we have the followings:

Corollary 5.1. The tangent plane turns evidently through $180^{\circ}$ along a time-like ruling (or time-like directrix) non-developable time-like ruled surface $M$.

Remark 5.1. Chales theorem is valid for a time-like (space-like) non-developable ruled surface. Hence, as an immediate result we have the following Theorem:

Theorem 5.1. Let $M$ be a non developable space-like ruled surface in $R_{1}^{3}$ and $\phi$ be the hyperbolic angle between the normal vector at the point of a ruling and the normal vector at the striction point of this ruling. Then tanh $\phi$ is proportional to the distance between these points and the coefficient of proportionality is the inverse of the distribution parameter.

\section{REFERENCES}

[1] W. Blacshke, Differential geometry, Istanbul University, No.433, 1949.

[2] H. W. Guggenheimer, Differential geometry, Mc Graw-Hill Book Company, 1963.

[3] H. H. Hacisalihoğlu, On the pitch of a closed ruled surface, Mechanism and Machine Theory, 7 (1972), 291-305.

[4] Ö. Köse, Contributions to the theory of integral invariants of a closed ruled surfaces, Mechanism and Machine Theory, 32 (1997), 261-277.

[5] A. Sarığlugil, A. Tutar, On ruled surfaces in Euclidean space E $E^{3}$, Int. J. Contemp. Math. Sci., 1, 2 (2007), 1-11.

[6] A. Turgut, H. H. Hacisalihoğlu, On the distribution parameter of time-like ruled surfaces in the Minkowski 3-Space, Far East J. Math. Sci., 2, 5 (1997), 321-328.

[7] A. Turgut, H. H. Hacısalihoğlu, Time-like ruled surfaces in the Minkowski 3-space-II, 1, 22 (1998), 33-46.

[8] B. O'neill, Semi-Riemannian geometry, Academic Press, New York-London, 1983.

[9] H. H. Uğurlu, A. Çalışkan, Frenet and Darboux vectors of a time-like curve on a time-like ruled surface, L. Spil Science Congress, September 4-5, Manisa, 1995.

[10] Y. Yayl, E. Özyılmaz, On the closed space-like developable ruled surface, Hadronic Journal, 23 (2000), 439-456.

[11] Y. Yaylı, E. Özyllmaz, On the closed motions and space-like ruled surfaces, Commun. Fac. Univ. Ankara Series A1, 49 (2000), 49-58. 